\title{
Pressure signature of forearm as predictor of grip force
}

\author{
Michael Wininger, BS; ${ }^{{ }^{*}}$ Nam-Hun Kim, PhD; ${ }^{1-2}$ William Craelius, PhD $^{\mathbf{1}}$ \\ ${ }^{1}$ Department of Biomedical Engineering, Rutgers, The State University of New Jersey, Piscataway, NJ; ${ }^{2}$ Department of \\ Physical Medicine and Rehabilitation, University of Medicine and Dentistry of New Jersey, Piscataway, NJ
}

\begin{abstract}
We studied the relationship between grip force and external forearm pressure in nondisabled subjects using force myography (FMG). FMG uses a sensorized cuff surrounding the forearm to register the distributed mechanical force, detecting pressure on the sensors generated by the volumetric changes of the underlying musculo-tendinous complex. Each of nine nondisabled subjects donned the FMG cuff and applied grip forces to a cylindrical dynamometer; grip forces ranged from $0 \%$ to $100 \%$ of the subjects' maximum voluntary contraction. The cuff was positioned with seven force sensors located on both the anterior and posterior surfaces of the proximal forearm, but no attempt was made to match sensor placement with particular muscles or sites. Grip prediction was simply encoded as the rectified sum of the FMG sensor outputs. During grip and release cycles, the FMG waveforms of each subject correlated closely with his or her force waveforms ( $r>$ 0.89). FMG also correlated highly with the timing of grip onset and release (intraclass correlation coefficient $(\operatorname{ICC}(\mathrm{A}, 2))=$ $0.99)$ and time to peak $(\operatorname{ICC}(\mathrm{A}, 2)=0.91)$, with negligible lag. These results demonstrate that when applied to the forearm, FMG represents a grip force signature that may be useful for near-real-time proportional control of upper-limb prosthetic devices.
\end{abstract}

Key words: control, feedback, force myography, forearm, grasp, grip force, hand, prosthesis, rehabilitation, sensors, upper-limb.

\section{INTRODUCTION}

Fundamental to human manual dexterity is the ability to precisely control the forces applied by the hand in proportion to the requirements of the task and/or object.
Restoring this ability to upper-limb amputees requires dynamic registration of the neuromuscular volition and faithful reproduction of the articulations encoded for the absent hand. This biomimetic paradigm necessitates both an interface for the extraction of neuromotor signatures from within the residuum [1-5] and a high-fidelity grip force control for proportional grasping in near real time [2,6-12]. The goal of this study was to critically evaluate force myography (FMG) as a representation of dynamic grip force for proportional control.

FMG registers the radially directed muscle pressures relating to the force produced by the contractile components as well as the corresponding series and parallel elastic components within the limb. Volitions concerning the hand, such as individual finger flexions, are encoded in the forearm FMG as characteristic force images that can be readily decoded for multifinger biomimetic control of robotic and virtual hands by amputees [1,13-16]. To date, FMG is the only neuromuscular imaging modality

Abbreviations: ANOVA = analysis of variance, $\mathrm{EMG}=$ electromyography, FMG = force myography, FSR = force-sensitive resistor, GFD = grip force dynamometer, ICC = intraclass correlation coefficient, $\mathrm{MVC}=$ maximum voluntary contraction, NIDRR = National Institute on Disability and Rehabilitation Research, RMS = root-mean-square, VR = variance ratio.

* Address all correspondence to Michael Wininger, BS; Department of Biomedical Engineering, Rutgers, The State University of New Jersey, 599 Taylor Rd, Piscataway, NJ 08854; 732-445-1178; fax: 732-445-2369.

Email: wininger@eden.rutgers.edu

DOI: 10.1682/JRRD.2007.11.0187 
that has demonstrated simultaneous multifunctional and multiple degrees-of-freedom control of prosthetic hands [13-15,17], including multifinger operation by amputees. Previous results with amputees suggested the potential of FMG as a register of force magnitude volition [13]. Herein, we directly test its effectiveness in predicting grip force in nondisabled limbs.

Our main aim was to establish the reliability of forearm FMG as a predictor of grip force generated in repetitive grasping tasks. We compared the temporal landmarks of grip onset, grip cessation, and time to maximum voluntary contraction (MVC) and assessed the within-subject variability of FMG and grip force traces. These comparisons directly assessed the suitability of forearm pressure maps as a proxy for grasp activities. We report that the dynamics of grasping tasks in nondisabled subjects can be accurately predicted by the summed and rectified FMG signals generated from the surface pressure signatures of the forearm.

\section{METHODS}

Nine nondisabled human volunteers performed a series of cyclic grasping tasks while data records of their forearm musculatures and handgrip forces were simultaneously recorded by FMG and grip force dynamometry, respectively.

\section{Force Myography Cuff}

A myokinetic FMG cuff (Figure 1) [18] was designed to measure mechanical activity of the extrinsic hand muscles in the forearm as described elsewhere [16]. The cuff consisted of an array of 14 force-sensitive resistors (FSRs) (1.4 cm diameter, model 402, Interlink Electronics; Carpenteria, California) placed at 7 anterior and 7 posterior loci within a standard forearm/wrist orthosis (Orthobionics, Inc; Dallas, Texas) that covered primarily the mid-to-proximal surface of the forearm. Though a securing strap was wrapped around the thumb, it did not affect grasping movement. Voltage output from the sensors was obtained from a half-bridge circuit with an FSR sensor and a $5 \mathrm{k} \Omega$ fixed resistor.

The FSR array reports changes in limb muscle activity as a change in voltage due to the expansion or contraction of the muscle belly radius during shortening or lengthening, which alters the pressure normal to the interfacing sensor. Sensor signals were acquired with 12-bit

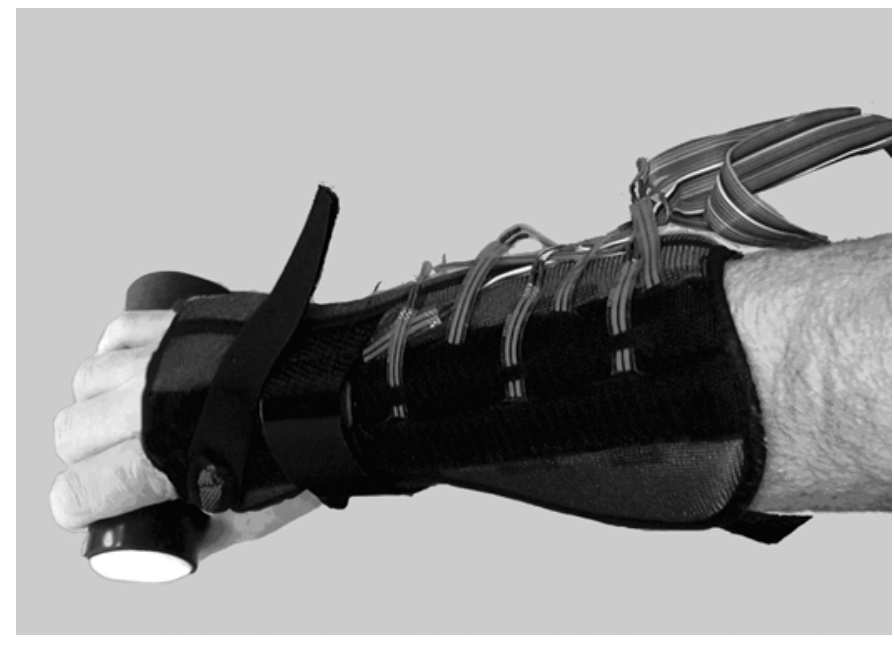

Figure 1.

Grip force dynamometer and force myography sensors. Sensors primarily detect proximal forearm musculature.

accuracy by an external data acquisition board (NI-DAQUSB-6008, National Instruments, Corp; Austin, Texas) and subsequently routed to LabVIEW (National Instruments, Corp), which provided the online processing as well as real-time visual feedback for target tracking.

The cuff was donned on the arm with a baseline pressure of $\sim 5$ percent full-scale output of the sensors, representing $25 \mathrm{mmHg}$, which was subtracted before each test (see "Signal Processing" section). Overall FMG response was calculated as mean square deviation from each sensor's baseline value (Equation 1) and stored using LabVIEW.

\section{Grip Dynamometry}

The custom grip force dynamometer (GFD) (Figure 1) was constructed as described elsewhere [18]. The GFD consisted of a polyvinyl chloride cylinder $(4 \mathrm{~cm}$ diameter $\times$ $10 \mathrm{~cm}$ long) onto which four strip FSRs (model 408, Interlink Electronics) were equidistantly placed along the entire length. The grip shape and orientation of the sensors were designed to maximize the contact between the sensors and the fingers, palm, and thumb. Voltage output of the quadrupolar parallel array of FSRs was obtained with half-bridge circuitry with a single $5 \mathrm{k} \Omega$ resistor. Data were acquired as described previously for the cuff.

\section{Signal Processing}

Raw instrument sensor data, $R^{\text {Inst }}$, were adjusted for baseline, $b^{\text {Inst }}$, on each sensor track acquired during 
calibration (Inst $=$ GFD or FMG) and condensed to a single track in $S^{\text {Inst }}$ a root-mean-square (RMS) rectification by

$$
S_{i}^{\text {Inst }}=\sqrt{\frac{1}{N^{\text {Inst }}} \sum_{\delta=1}^{N^{\text {Inst }}}\left(R_{i \delta}^{\text {Inst }}-b_{\delta}^{\text {Inst }}\right)^{2}},
$$

where the number of sensors $N^{\mathrm{FMG}}=7$ and $N^{\mathrm{GFD}}=4$ and $i$ is the data sample index. FMG output was simply represented as $S_{i}$ FMG without further processing. GFD signals, however, were linearized (since the raw output of the FSRs is nonlinear) by

$$
R_{\delta}^{\mathrm{GFD}}=\mathcal{L}^{-1}\left\{G_{\delta}(s) * Y(s)\right\},
$$

where $G_{\delta}(s)$ is the Laplace transform of individual GFD sensor outputs, $\mathcal{L}^{-1}$ is the inverse Laplace transform, and $Y(s)$ is the empirically determined third-order transfer function found in the GFD linearization procedure

$$
Y(s)=\frac{0.09 s^{3}+2.70 s^{2}-6.00 s+35.64}{s^{4}},
$$

where $s$ is the representation of the time-domain signal in complex angular frequency domain. In this way, the grip force display was linearized. Both the rectified FMG and linearized GFD signals were low-pass filtered with a second-order Butterworth filter with a $4 \mathrm{~Hz}$ cutoff. Linearized GFD force signatures (scaled to \% MVC during the preprotocol calibration) were delivered to the display screen for real-time visual feedback proportional to user grip forces (Figure 2).

\section{Subject Protocols}

Nine nondisabled subjects (8 male, 1 female; $23.9 \pm$ 5.5 years old; all right-side dominant; of moderate to athletic fitness; and with body mass index ranging from 18.9 to 24.4) were recruited for the study. (All data are presented as mean \pm standard deviation unless otherwise noted.) Subjects were selected to reflect the expected demography of a typical user population, such as returning service personnel. All subjects provided informed written consent, with the protocol approved by the institutional review board of Rutgers. Each subject donned the FMG cuff and grasped the grip dynamometer with his or her right hand while sitting comfortably in front of the

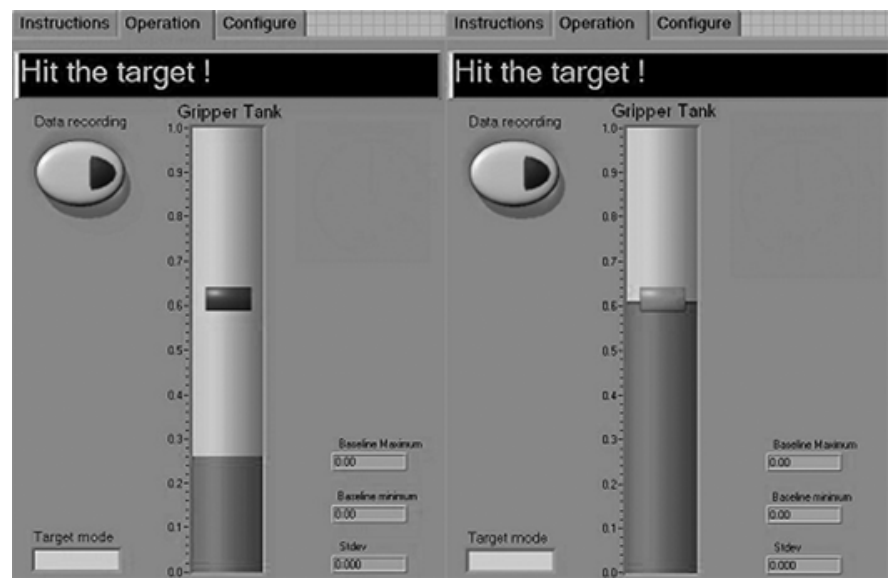

Figure 2.

Interface screen shot (LabVIEW) showing feedback of moderate activation (left) and target achievement (right).

computer screen (19 in. liquid crystal display). The subject's elbow rested on a table, with the unsupported forearm suspended in the air to prevent perturbation to the FMG sensors. All subjects maintained their arm in a neutral position throughout the protocol. The screen displayed a single tank that provided visual feedback in the target tracking tasks, as shown in Figure 2. Prior to testing, we corrected the display grip force for baseline and scaled the data for maximum force with the following protocol. Subjects grasped the GFD by wrapping their fingers and thumb around it with their wrist in neutral position and completely relaxed, with force just sufficient to hold the GFD. They were then asked to maximally grasp with their full cylindrical grip force to establish their MVC. These two raw output values were used to scale the visual feedback tank display from 0 to 100 percent MVC. The FMG was calibrated simultaneously, according to the voltages produced at the cuff sensors; no visual feedback was provided for this calibration.

To test the correlation between grip force and forearm FMG, we asked subjects to perform repeated cycles of grasp and release, with or without a matching target. For the targeting protocols, we asked subjects to match their grip force output to a moving visual target that consisted of a large red mark within a blue tank. The following specific protocols were administered in random order: (1) tracking of sinusoids at a slow pace $(0.05 \mathrm{~Hz})$ for $60 \mathrm{~s}$, followed by variable rest periods (3 sets); (2) tracking of sinusoids at a fast pace $(0.1 \mathrm{~Hz})$ for $60 \mathrm{~s}$, followed by variable rest periods (3 sets); and (3) self-paced sinusoidal patterns without visual feedback of force (30 s/set) (3 sets). 
In addition, we asked subjects to produce a sudden force to 50 percent MVC and hold it for $20 \mathrm{~s}$. During protocols 1 and 2, visual feedback came from the GFD only. The overall experimental setup is depicted in Figure 3.

\section{Waveform Comparisons}

To compare GFD and FMG waveforms, we first separated the processed signals from each instrument, $S_{i}^{\text {Inst }}$, into their constituent grasp cycles. We did this in one of two ways: a supervised method, where fiducial points extracted from the target wave were used to separate grip cycles from FMG and GFD, and an unsupervised method that separated the cycles using only the intrinsic waveform features of GFD and FMG. The supervised method is primarily a means by which an identical window can be imposed over both signals for waveform comparisons. In both methods, a cycle was defined by consecutive local minima determined by manual thresholding performed with a custom program written in MATLAB ${ }^{\circledR}$ (The MathWorks, Inc; Natick, Massachusetts).

FMG and GFD waveforms were first compared in a cyclewise interinstrument comparison via the Pearson product moment correlation coefficient $(r)$ given by

$$
r=\frac{\operatorname{cov}\left(S^{\mathrm{FMG}}, S^{\mathrm{GFD}}\right)}{\sqrt{\operatorname{cov}\left(S^{\mathrm{FMG}}, S^{\mathrm{FMG}}\right)} \sqrt{\operatorname{cov}\left(S^{\mathrm{GFD}}, S^{\mathrm{GFD}}\right)}},
$$

where the correlation substrates $(S)$ were the processed one-dimensional signal waveforms from the FMG and GFD and cov the covariance.

Using grip data extracted from a supervised separation and temporally normalized by an inline MATLAB interpolation function, we assessed between-test repeatability of FMG and GFD signals. Peak amplitudes of each cycle were also normalized to unity since waveform morphology was the only parameter of interest. We used the variance ratio (VR) to assess the grip-to-grip variability as rendered by each instrument [19]:

$$
\mathrm{VR}=\frac{\sum_{i=1}^{k} \sum_{j=1}^{n} \frac{\left(X_{i j}-\hat{X}_{i}\right)^{2}}{k(n-1)}}{\sum_{i=1}^{k} \sum_{j=1}^{n} \frac{\left(X_{i j}-\overline{\bar{X}}\right)^{2}}{k n-1}}
$$

where $X$ is the array of $j$ temporally normalized signals; $\hat{X}_{i}$ is the average value of the $n$ waveforms at time-point $i$, running to total time $k$; and $\overline{\bar{X}}$ is the data set grand mean. For perfectly identical signals, VR is equal to 0 , and for completely unrelated or random signals, VR is equal to 1 . In a multiple-repetition cyclic grasping task, the VR can determine signal waveform similarity for all repetitions (not just between two, as with the correlation coefficient); temporal shifts are ignored by uniform cycle extraction.

To estimate temporal correlations, we extracted onset and offset times for each grip cycle from processed and filtered data sets as described previously. The time-tomaximum effort was determined as the time of maximum signal strength between each set of onset-offset times. Temporal landmarks were extracted with a completely unsupervised process, with fixed thresholds for the output GFD and FMG waves and without reference to the target.

Temporal landmarks from both instruments were compared via the intraclass correlation coefficient (ICC), which quantifies the proportion of variance attributable to the objects of measurement, i.e., the relationship between variables sharing both metric and variance. Two versions of the ICC, each based on a two-way analysis of variance (ANOVA), were employed: consistency, $\operatorname{ICC}(\mathrm{C}, 2)$, and agreement, ICC(A,2). Interrater differences between GFD and FMG were estimated by

$$
\mathrm{ICC}(\mathrm{C}, 2)=\frac{\mathrm{MSR}-\mathrm{MSE}}{\mathrm{MSR}+(\kappa-1) \cdot \mathrm{MSE}}
$$

and

$$
\operatorname{ICC}(\mathrm{A}, 2)=\frac{\mathrm{MSR}-\mathrm{MSE}}{\mathrm{MSR}+(\kappa-1) \cdot \mathrm{MSE}+\frac{\kappa}{v}(\mathrm{MSC}-\mathrm{MSE})},
$$

where MSR and MSE are the mean squared errors of row variables and error obtained from ANOVA, respectively; $k$ is the number of measurable objects (i.e., instruments, FMG and GFD); and $n$ is the number of measurement instances (i.e., grasp cycles, which varies from subject to subject and test to test) [20]. These two-way models were selected over the one-way random effects model, ICC(A,1), because the two measurement modalities constitute separable sources of variability. ICC values range from 0 to 1 , representing completely uncorrelated and identical data sets, respectively. 


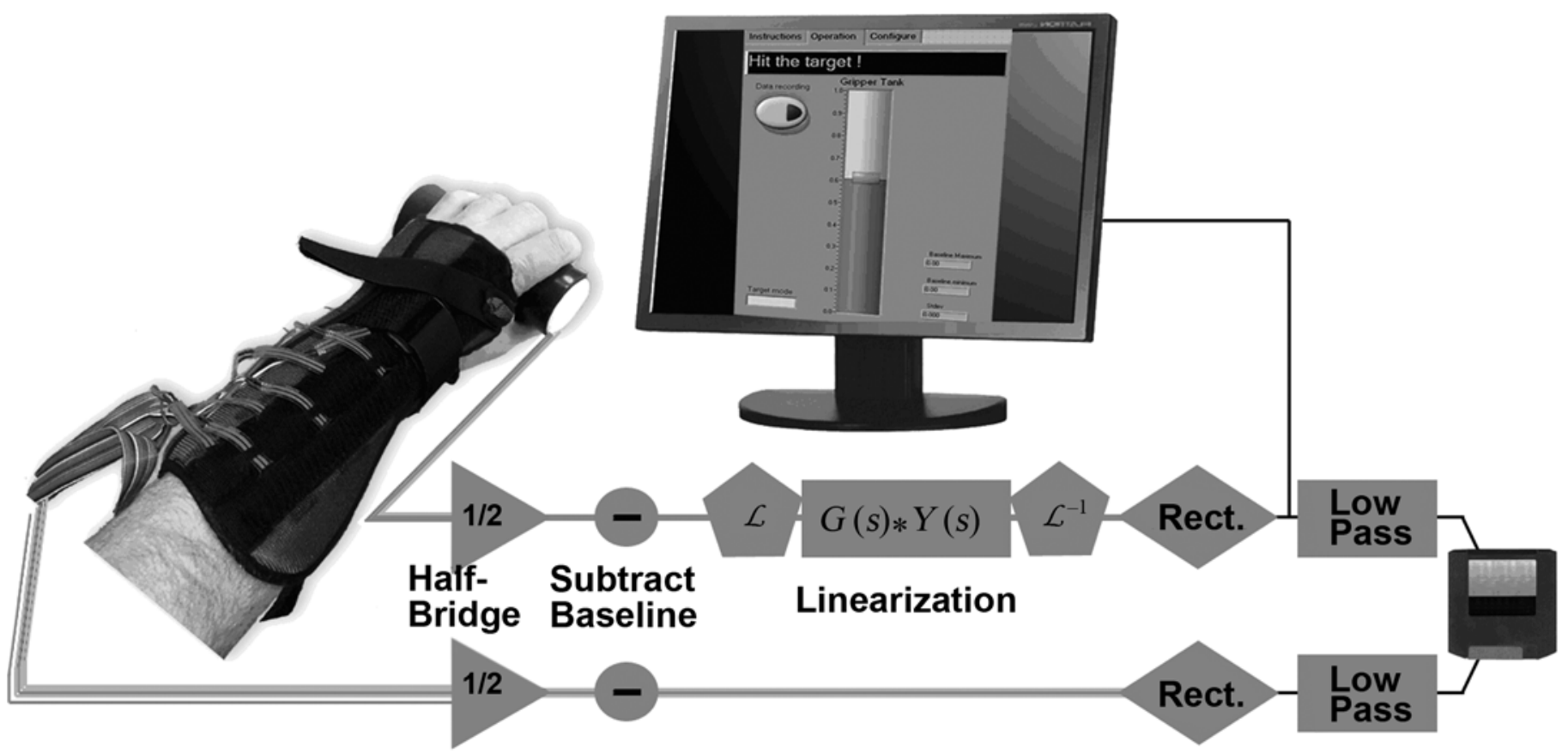

Figure 3.

Instrumentation and signal processing flow chart. $G(s)$ and $Y(s)$ denote the convolution of the Laplace-transformed grip force dynamometer sensor outputs onto Fourier-domain representation of linearization equation (Equation 3). These signals were transformed by Laplacian operator (denoted by $\mathcal{L}$ ) and converted back into time domain by inverse Laplace $\left(\mathcal{L}^{-1}\right)$. Low Pass = second order Butterworth low-pass filter with $4 \mathrm{~Hz}$ cutoff, Rect. $=$ rectified.

A final analysis was performed on the linear relationship of these temporal landmarks. We simultaneously compared timing data from all protocols via a crosscorrelation coefficient. We evaluated slopes and intercepts for their deviations from 1 and 0 , respectively.

\section{RESULTS}

\section{Waveform Correlation}

Data from a typical subject executing two complete cycles for the target-tracking protocols are shown in Figure 4. Note the close correspondence of the FMG waves to those of the GFD in terms of timing of onset, peak, and offset as well as in overall shape and relative amplitudes. Many sudden spikes in the waveforms seen in the grip force signal appear to be mirrored in the FMG signal. To ascertain the stability of the FMG signal during sustained grips, we asked each subject to grip to 50 percent MVC and hold the grip for 20 seconds. A sample trace is presented in Figure 4(c). Note that the FMG signal followed closely, without visible lag, relative to the onset and cessation of GFD and remained stable throughout the duration of the grasp.

Each of the nine subjects performed an average of $34.2 \pm 13.5$ grasp/release cycles, executing two sinusoidal tasks and one self-paced task. The mean correlation coefficient between FMG and GFD for all cycles $(N=$ 391) was $0.89 \pm 0.05$, as shown in Table 1. A one-way ANOVA did not reveal a significant difference in correlation coefficients among tasks.

\section{Waveform Repeatability}

The VR (Equation 5) reports the summed variability among all grip cycles within the data sets generated by both instruments. VRs for FMG, GFD, and the computergenerated target are listed in Table 2 . The cycle-to-cycle VR of GFD at the high targeting speed was significantly lower than that of FMG ( $p<0.001)$; however for slow targets, no significant difference was found between the modalities from one-way ANOVA on group means. GFD for slow targeting had a very low VR $(0.082 \pm 0.091)$ and was close to that of the target variability $(0.078 \pm 0.113)$, indicating that for slow tasks, signal repeatability of both 
(a)

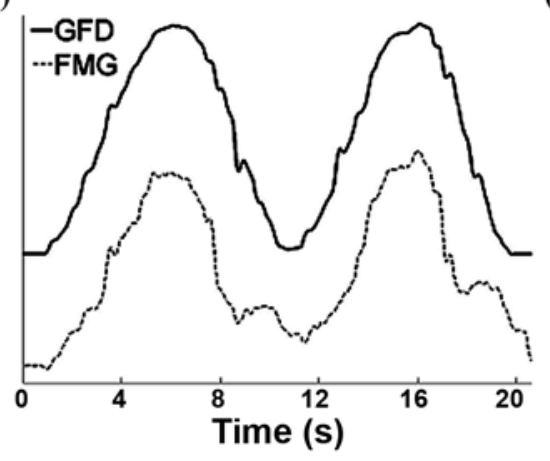

(b)

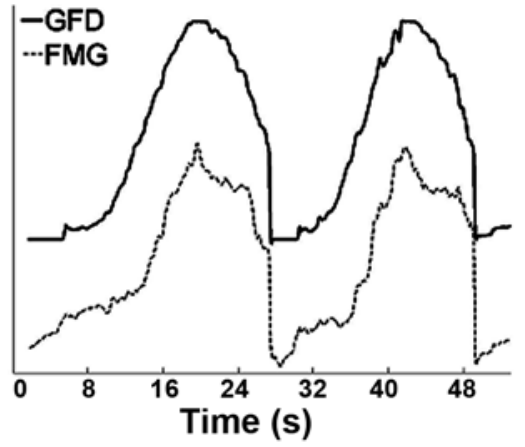

(c)

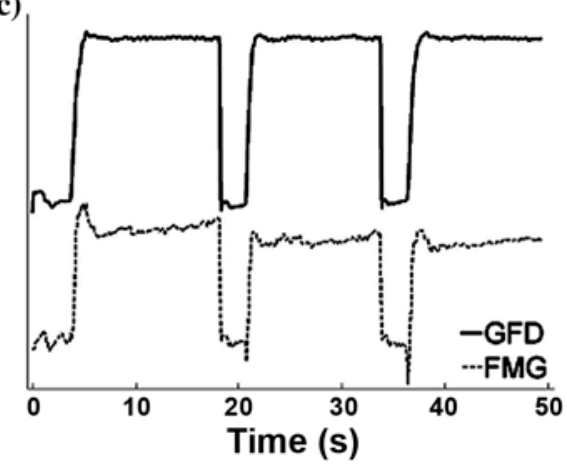

Figure 4.

Sample records of grip force dynamometer (GFD) and force myography (FMG) from protocols (a) 1 and (b) 2 (2 cycles) (see main text) and (c) isometric grasp sustained at 50\% maximum voluntary contraction for $10 \mathrm{~s}$ (3 cycles).

Table 1.

Waveform correlation results (mean \pm SD) comparing forearm pressure signals detected by force myography to isometric power grip force output detected by grip force dynamometer. Subject averages were $r=0.89 \pm 0.05, r_{\min }=0.68, r_{\max }=0.97, \mu_{\text {group }}=34.22 \pm 13.48$, Min $_{\text {group }}=17$, and Max $_{\text {group }}=54$.

\begin{tabular}{lcr}
\hline \multicolumn{1}{c}{ Task } & $\begin{array}{c}\text { Task-Specific } \\
\text { Correlation }\end{array}$ & $\boldsymbol{\mu}_{\text {reps }}$ \\
\hline Self-Paced & $0.90 \pm 0.05$ & $16.22 \pm 5.86$ \\
Slow Target & $0.90 \pm 0.05$ & $6.33 \pm 4.09$ \\
Fast Target & $0.86 \pm 0.10$ & $11.67 \pm 6.96$ \\
\hline Max $=$ maximum, Min $=$ minimum, SD = standard deviation, $\mu_{\text {group }}=$ grand \\
mean \pm SD, $\mu_{\text {reps }}=$ mean \pm SD per subject. \\
\hline \hline
\end{tabular}

Table 2.

Between-subject variance ratios (mean \pm standard deviation) for targeted grasping tasks.

\begin{tabular}{lccc}
\multicolumn{1}{c}{ Task } & Target & Hand & Forearm \\
\hline Slow Target & $0.078 \pm 0.113$ & $0.082 \pm 0.091$ & $0.188 \pm 0.164$ \\
Fast Target & $0.005 \pm 0.005$ & $0.027 \pm 0.015$ & $0.085 \pm 0.033$ \\
\hline \hline
\end{tabular}

instruments approximated that of the computer. VRs for both GFD and FMG were lower for $0.1 \mathrm{~Hz}$ targeting.

\section{Temporal Landmark Correlation}

The ICC values for each protocol are presented in Table 3. ICC(C,2) and ICC(A,2) values in excess of 0.99 indicate a high correlation between the two measurement systems in terms of onset and offset. Time to peak also correlated well (ICC $\geq 0.95$ ).

The relationship between temporal landmarks for FMG and GFD is graphically shown in Figure 5. Analysis of regression parameters from the Pearson product moment correlation revealed a slope of $m=0.98 \pm 0.05$ and intercept of $b=0.01 \pm 0.02$; Student's $t$-test did not reveal a significant departure from $m=1$ and $b=0$ at the $p<0.05$ level. Thus, timing by FMG directly corresponds to timing by GFD, with negligible delay.

\section{DISCUSSION}

\section{Validity of Study}

This article studied forearm pressure signature as a predictor of grip force. Although we report on only nondisabled subjects, the results may apply to the transradial amputee population, since previous studies have shown that the amplitude of single-finger volitions can by extracted via FMG [15,17]. Test conditions were limited to the arm and wrist in a fixed neutral position in the horizontal plane. This pose simulates a common working condition, and moderate departures from this posture are unlikely to significantly alter the waveform characteristics of the FMG signal. Moreover, in prosthetic applications, pronation/supination of the wrist is not an issue, and the FMG interface can adapt well to different arm conditions by calibration upon donning [14-15].

Although it would be valuable, a direct comparison between FMG and electromyography (EMG) is beyond the scope of this article, and further work is needed to establish the utility of FMG as a control modality. Specifically, FMG should be tested under conditions of variable loads, ballistic motions, and increasingly dynamic activities. Additionally, whereas FMG has demonstrated the capacity to resolve forearm pressure signatures during 
Table 3.

Intraclass correlation coefficients (ICCs) comparing force myography and grip force dynamometer for grasping task temporal landmarks (onset, offset, time-to-maximum). ICC(C,2) = rater consistency and $\operatorname{ICC}(\mathrm{A}, 2)=$ rater absolute agreement. Data presented as mean \pm standard deviation.

\begin{tabular}{|c|c|c|c|c|}
\hline \multirow{2}{*}{ Task } & \multicolumn{2}{|c|}{ Onset \& Offset } & \multicolumn{2}{|c|}{ Time-to-Maximum } \\
\hline & ICC(C,2) & $\operatorname{ICC}(A, 2)$ & ICC(C,2) & $\operatorname{ICC}(A, 2)$ \\
\hline Self-Paced & $0.99 \pm 0.02$ & $0.99 \pm 0.001$ & $0.95 \pm 0.04$ & $0.91 \pm 0.10$ \\
\hline Slow Target & $0.99 \pm 0.01$ & $0.99 \pm 0.001$ & $0.97 \pm 0.03$ & $0.95 \pm 0.05$ \\
\hline Fast Target & $0.99 \pm 0.01$ & $0.99 \pm 0.001$ & $0.97 \pm 0.03$ & $0.95 \pm 0.05$ \\
\hline
\end{tabular}

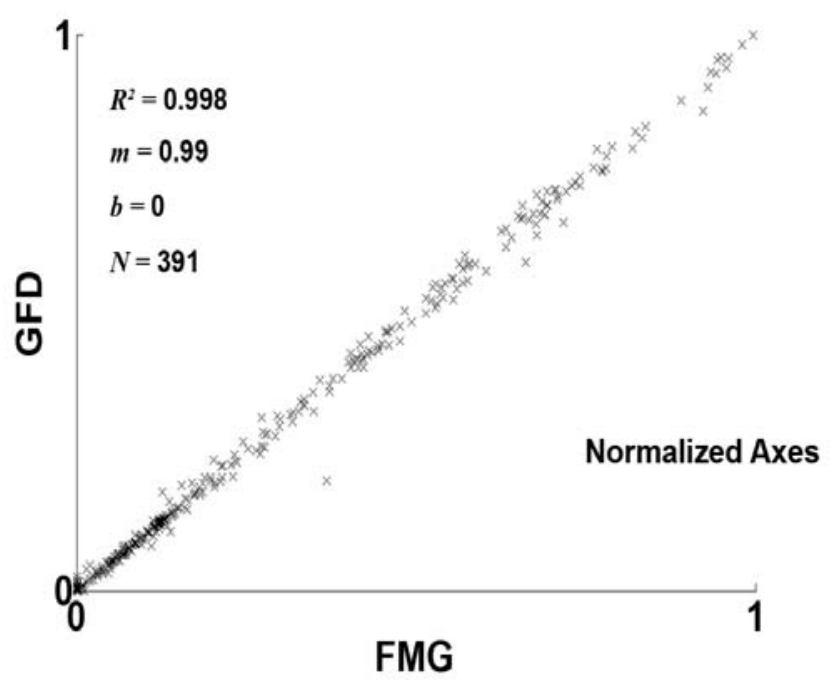

Figure 5.

Comprehensive plot of linear regression to temporal landmarks of force myography (FMG) detection of forearm muscle pressure vs grip force dynamometer (GFD) detection of grasp force. Across all protocols, subjects exhibited linear slope of $m=0.99$ and intercept of $b<0.001$, fitting 391 grasps. Scale normalized for clarity.

several grip types (such as precision pinch, mouse-click, and cylindrical grasp) [21], it remains to be established whether these postures are equally predictive of their respective force outputs at the hand. Future studies should also include assessments of longitudinal variation, but FMG interpretation is expected to be relatively insensitive to both daily and long-term changes in the residual limb.

The ability of FMG to resolve hand postures and individual finger motions has previously been demonstrated in transradial amputees, some of whom were $>15$ years postamputation and had relatively poor residua [14-15,17]. More recently, it has been shown that six different grip types can be classified with considerable accuracy when FMG is used to measure intact forearms
[21]. The results presented herein demonstrate the viability of FMG as a proportional control system in prosthetic applications.

\section{Grip Force Prediction}

We performed several interrater analyses in order to assess the correspondence between FMG of the forearm and the grip force produced during cyclic and sustained grasping tasks. Point-to-point waveform correlation of signals from the FMG and GFD yielded the most powerful rating of compatibility between measurement modalities. Analysis of 391 cycles of cyclic grasp and release produced by nine subjects yielded a mean cross-correlation of $0.89 \pm 0.05$ between GFD and FMG waveforms (Table 1), with no significant differences between tasks. Thus, FMG accurately represents grip force continuously over its entire range and is sensitive to minute adjustments in timing and magnitude of force application. These characteristics are crucially relevant to the needs of users of upper-limb prostheses: a volitional control paradigm with a high-fidelity real-time correspondence to end-effector activity is necessary for user safety, compliance, and reliability in device operation. We should note that though the waveform correspondence between GFD and FMG was generally excellent throughout the grasp and release cycles, the FMG displayed overshoot and undershoot during the stepwise grips that was much larger than that seen in the GFD signal (Figure 4). This apparent amplification of force transients by FMG may be due to thixotropic properties of the forearm and could readily be filtered if needed.

Since cross-correlation is insensitive to possible phase shifts between signals, we evaluated the accuracy of FMG in timing of grasp forces using the ICCs. As shown in Table 3, ICC $(\mathrm{A}, 2)$ reports the absolute agreement and ICC $(C, 2)$ reports consistency of the FMG's respective detection of temporal landmarks of grasp activity including onset, cessation, and peak grip force. ICCs for 
timing of grip onset, peak, and release all exceeded 0.90 (Table 3), demonstrating the fidelity of FMG of the forearm in predicting changes in grasp forces. These ICC values compare favorably to those reported for $M$-wave EMG normalization factors that ranged from 0.95 to 0.98 [22]. Additionally, temporal landmarks extracted from FMG and GFD data records were evaluated for their slope and intercept parameters. The regression fits were extremely close $\left(R^{2}>0.99\right)$ and yielded slopes and intercepts that were not significantly different from $m=1$ and $b=0$. Thus, over the course of a session, the timing of even subtle variations in grasp forces remains highly predictable via FMG, with negligible lag.

Waveform repeatability is critical to the reliability of any encode/decode system, and its accuracy can be efficiently assessed across any number of repetitions using the VR [19]. Across successive grasp and release cycles, each subject produced relatively invariant waveforms in their FMG signals, as quantified by their extremely low VRs (Table 2). Testing approximately 34 repeat FMG waveforms during grasp and release by nine subjects, we found that VRs for FMG averaged 0.085 and 0.188 for fast and slow tracking, respectively (Table 2). These VRs indicate a high degree of repeatability and compare favorably with those of EMG. For example, EMG $M$ waves from the biceps brachii were judged to be "very reproducible" with a VR of $0.24 \pm 0.17$ [23]. Also, comparison of the surface EMG of musculus flexor digitorum supericialis between skilled archers and nonarchers yielded VRs of 0.08 and 0.70 , respectively [24]. FMG repeatability tested in untrained subjects thus parallels that of EMG in highly trained individual muscles.

Although the ability of subjects' hands to track the target force was not explicitly analyzed, we found it to be highly accurate based on the low VRs for GFD, especially for the slow cycle (0.082), which did not differ significantly from that of the computer target VR (0.078). VRs for FMG were two to three times greater than those of GFD, indicating the occurrence of some signal degradation between the forearm muscles and the joints.

\section{Force Myography as Control Modality}

Proportional control of grip force is a feature highly desired by users of upper-limb prostheses, but presently, few and limited options are available to them. The ProController ${ }^{\circledR}$ (Motion Control, Inc; Salt Lake City, Utah) offers the most advanced force-controlled prosthesis through use of EMG levels and force feedback for con- trol, but its limitation to a few discrete states has not significantly improved since its introduction in the 1980s [25]. Many studies have demonstrated the potential of EMG models and advanced signal processing to predict grip force, but none has demonstrated that these modalities have been practically implemented in a prosthesis [23,26-31]. However, we expect that the inherently low frequency information associated with FMG measurement of superficial changes in muscle conformation will more precisely render subtle changes in grasp dynamics.

The most obvious difference between FMG and myoelectric control is the sensing of mechanical rather than electrical signals. Mechanical output of muscles represents a low-bandwidth and degraded version of the neuroelectrical signals and cannot resolve activities of individual muscles. FMG, after the processing used herein, is rather analogous to the average rectified values of surface EMG and the RMS-based EMG processing described by Basmajian and De Luca [32]. Surface EMG may be considered a structure-based model, reductionally representing the system's important elements [33], whereas FMG is a more phenomenological model, based on its direct correlations and resemblance to grip force.

To incorporate FMG into a versatile prosthetic controller, researchers must establish its utility beyond the laboratory and answer several practical questions. These include reliability and durability of the sensor sleeves, the ease of donning for users, ease of calibration, and compatibility with controller interfaces. Preliminary trials with amputees have demonstrated that donning and calibration of FMG are relatively simple, that its sensitivity is not seriously compromised by the wearing of a protective stocking, and that it works well with residua with severely damaged tissue [13]. A crucial component that is still lacking is an electronic interface with sufficient analog inputs that are compatible with diverse sensors to enable multidimensional control. The current Defense Advanced Research Projects Agency initiative, "Revolutionizing upper-limb prosthetics," [34] will no doubt stimulate progress toward this end.

\section{CONCLUSIONS}

Our results indicate that the grip force signature provided by FMG may be a useful alternative to EMG as a noninvasive prosthetic interface for proportional grip control. The FMG, using a simple summation of pressures recorded on the forearm, closely mirrors the grip force continuously from rest to 100 percent MVC. Point-by- 
point signal processing with minimal filtering makes real-time operation of FMG control a realistic paradigm. Since FMG does not depend on precise positioning of sensors on the limb, prosthetic users may daily don and doff the interface, without concern for precise positioning. The calibration process requires two measurements: baseline and maximum effort. We conclude that FMG of the forelimb accurately reflects grip force and may be useful in providing intuitive control of upper-limb prostheses.

\section{ACKNOWLEDGMENTS}

This material was the result of work supported by a U.S. Department of Education National Institute on Disability and Rehabilitation Research (NIDRR) Small Business Innovation Research Program Phase II Award (grants ED 84.305S and CFDA 84.133S) and a Rehabilitation Engineering Research Center grant from NIDRR in collaboration with the New Jersey Institute of Technology on "Improving early manipulation.” Fellowship support for Michael Wininger was provided by the National Science Foundation Integrative Graduate Education and Research Traineeship on "Integratively engineered biointerfaces” at Rutgers (grant DGE 033196, Prabhas Moghe, principal investigator).

The authors have declared that no competing interests exist.

\section{REFERENCES}

1. Craelius W. The bionic man: Restoring mobility. Science. 2002;295(5557):1018-21. [PMID: 11834819]

2. Kuiken TA, Lowery MM, Stoykov NS. The effect of subcutaneous fat on myoelectric signal amplitude and cross-talk. Prosthet Orthot Int. 2003;27(1):48-54. [PMID: 12812327]

3. Micera S, Carrozza MC, Beccai L, Vecchi F, Dario P. Hybrid bionic systems for the replacement of hand function. Proc IEEE. 2006;94(9):1752-62.

4. Lai JC, Schoen MP, Perez Gracia A, Naidu DS, Leung SW. Prosthetic devices: Challenges and implications of robotic implants and biological interfaces. Proc Inst Mech Eng [H]. 2007;221(2):173-83. [PMID: 17385571]

5. Carrozza MC, Cappiello G, Micera S, Edin BB, Beccai L, Cipriani C. Design of a cybernetic hand for perception and action. Biol Cybern. 2006;95(6):629-44. [PMID: 17149592]

6. Hermansson LM, Fisher AG, Bernspang B, Eliasson AC. Assessment of capacity for myoelectric control: A new
Rasch-built measure of prosthetic hand control. J Rehabil Med. 2005;37(3):166-71. [PMID: 16040474]

7. Chiou YH, Luh JJ, Chen SC, Lai JS, Kuo TS. The comparison of electromyographic pattern classifications with active and passive electrodes. Med Eng Phys. 2004;26(7):605-10. [PMID: 15271288]

8. Farrell TR, Weir RF. The optimal controller delay for myoelectric prostheses. IEEE Trans Neural Syst Rehabil Eng. 2007;15(1):111-18. [PMID: 17436883]

9. Farry KA, Walker ID, Baraniuk RG. Myoelectric teleoperation of a complex robotic hand. IEEE Trans Robot Autom. 1996;12(5):775-88.

10. Soares A, Andrade A, Lamounier E, Carrijo R. The development of a virtual myoelectric prosthesis controlled by an EMG pattern recognition system based on neural networks. J Intell Inform Syst. 2003;21(2):127-41.

11. Englehart K, Hudgins B. A robust, real-time control scheme for multifunction myoelectric control. IEEE Trans Biomed Eng. 2003;50(7):848-54. [PMID: 12848352]

12. Boostani R, Moradi MH. Evaluation of the forearm EMG signal features for the control of a prosthetic hand. Physiol Meas. 2003;24(2):309-19. [PMID: 12812417]

13. Abboudi RL, Glass CA, Newby NA, Flint JA, Craelius W. A biomimetic controller for a multifinger prosthesis. IEEE Trans Rehabil Eng. 1999;7(2):121-29. [PMID: 10391581]

14. Curcie DJ, Flint JA, Craelius W. Biomimetic finger control by filtering of distributed forelimb pressures. IEEE Trans Neural Syst Rehabil Eng. 2001;9(1):69-75. [PMID: 11482365]

15. Phillips SL, Craelius W. Residual kinetic imaging: A versatile interface for prosthetic control. Robotica. 2005;23: 277-82.

16. Flint J, Kuttuva M, Burdea G, Craelius W. Manipulation practice for upper-limb amputees using virtual reality. Presence. 2004;14(2):175-82.

17. Kuttuva M, Burdea G, Flint J, Craelius W. Manipulation practice for upper-limb amputees using virtual reality. Presence. 2005;14(2):175-82.

18. Kim N-H, Wininger M, Craelius W. Predicting direct handgrip force from extrinsic muscle output with force myography (FMG) [abstract]. In: Proceedings of the American Society of Biomechanics Northeast Conference; 30-31 Mar 2007; University of Maryland (MD).

19. Hwang IS, Lee HM, Cherng RJ, Chen JJ. Electromyographic analysis of locomotion for healthy and hemiparetic subjects-Study of performance variability and rail effect on treadmill. Gait Posture. 2003;18(1):1-12. [PMID: 12855295]

20. McGraw KO, Wong SP. Forming inferences about some intraclass correlations coefficients. Psychol Methods. 1996; 1(4):30-46.

21. Yungher DC, W. Discriminating 6 grasps using force myography of the forearm [abstract]. In: Proceedings of the 
American Society of Biomechanics Northeast Conference; 30-31 Mar 2007; University of Maryland (MD).

22. Calder KM, Hall LA, Lester SM, Inglis JG, Gabriel DA. Reliability of the biceps brachii M-wave. J Neuroeng Rehabil. 2005;2:33. [PMID: 16332261]

23. Rainoldi A, Galardi G, Maderna L, Comi G, Lo Conte L, Merletti R. Repeatability of surface EMG variables during voluntary isometric contractions of the biceps brachii muscle. J Electromyogr Kinesiol. 1999;9(2):105-19. [PMID: 10098711]

24. Soylu AR, Ertan H, Korkusuz F. Archery performance level and repeatability of event-related EMG. Hum Mov Sci. 2006;25(6):767-74. [PMID: 16859789]

25. Sears HH, Shaperman J. Proportional myoelectric hand control: An evaluation. Am J Phys Med Rehabil. 1991; 70(1): 20-28. [PMID: 1994966]

26. Farina D, Merletti R, Enoka RM. The extraction of neural strategies from the surface EMG. J Appl Physiol. 2004; 96(4):1486-95. [PMID: 15016793]

27. Gordon KE, Ferris DP. Proportional myoelectric control of a virtual object to investigate human efferent control. Exp Brain Res. 2004;159(4):478-86. [PMID: 15258714]
28. Hoozemans MJ, Van Dieen JH. Prediction of handgrip forces using surface EMG of forearm muscles. J Electromyogr Kinesiol. 2005;15(4):358-66. [PMID: 15811606]

29. Keir PJ, Mogk JP. The development and validation of equations to predict grip force in the workplace: Contributions of muscle activity and posture. Ergonomics. 2005;48(10): 1243-59. [PMID: 16253943]

30. Mogk JP, Keir PJ. Prediction of forearm muscle activity during gripping. Ergonomics. 2006;49(11):1121-30. [PMID: 16950725]

31. Duque J, Masset D, Malchaire J. Evaluation of handgrip force from EMG measurements. Appl Ergon. 1995;26(1): 61-66. [PMID: 15677002]

32. Basmajian JD, De Luca CJ. Muscles alive: Their functions revealed by electromyography. 5th ed. Baltimore (MD): Williams and Wilkins; 1985.

33. Stegeman DF, Blok JH, Hermens HJ, Roeleveld K. Surface EMG models: Properties and applications. J Electromyogr Kinesiol. 2000;10(5):313-26. [PMID: 11018441]

34. Guinnessy P. DARPA joins industry, academia to build better prosthetic arms. Physics Today. 2006;59(9):24-25.

Submitted for publication November 13, 2007. Accepted in revised form March 18, 2008. 